\title{
LncRNA HOTAIR regulates anoikis-resistance capacity and spheroid formation of ovarian cancer cells by recruiting EZH2 and influencing H3K27 methylation
}

\author{
Zhuo-Ya DAI, Shuang-Mei JIN*, Hong-Qin LUO, Hong-Lian LENG, Jun-Dan FANG \\ Department of Gynecology and Obstetrics, People's Hospital of Bishan County, Chongqing, China \\ ${ }^{*}$ Correspondence: jinshuangmei_cq@yeah.net
}

Received November 12, 2020 / Accepted December 27, 2020

\begin{abstract}
This study aims to investigate the role of the long non-coding RNA (lncRNA) HOX transcript antisense RNA (HOTAIR) in the regulation of anoikis resistance of ovarian cancer cells, a prerequisite for metastasis and chemoresistance in ovarian cancer cells. Ovarian cancer SKOV3 cells were cultured in an ultra-low attachment system to establish an anoikis model. The relationship between cellular anoikis capability and HOTAIR expression level was studied by flow cytometry and RT-PCR. The ability of spheroid formation, migration, and invasion of the suspended cells was assessed following the knockdown of HOTAIR expression. The expression of EZH2, H3K27me3, representative targets of EZH2, and anoikis-related biomarkers was also detected. An increase in the duration of suspension culture time rendered the SKOV3 cells anoikis-resistant with a significantly lower apoptotic rate compared to the adherent cells. HOTAIR expression in the suspension cells increased significantly, while that in the adherent cells did not. Following small interfering RNA (siRNA)-mediated knockdown of HOTAIR expression, the abilities of anoikis resistance, migration, and invasion decreased in the suspension cells. Knockdown of HOTAIR levels also reduced the spheroid forming ability of the tumor cells in continuous suspension cultures. Moreover, EZH2 expression correlated with HOTAIR expression, thus regulating the expression of miR-193a and DOK2 via introducing H3K27me3. Western blot analysis of anoikis-related markers showed that N-cadherin, ZEB1, and TWIST1 were downregulated following inhibition of HOTAIR, while E-cadherin and ErbB3 were upregulated. In conclusion, HOTAIR enhances the anoikis resistance and spheroid forming ability of ovarian cancer cells by recruiting EZH2 and influencing H3K27 methylation that may contribute to migration, invasion, and chemoresistance of ovarian cancer cells.
\end{abstract}

Key words: anoikis, spheroid, ovarian cancer, HOX transcript antisense RNA, EZH2

Ovarian cancer is the most lethal gynecologic cancer with epithelial ovarian cancer accounting for $85-90 \%$ of all primary ovarian cancers [1]. Factors that contribute to the poor prognosis of ovarian cancer include failure of early detection, a high degree of pelvic and peritoneal metastasis, and inherent or acquired chemoresistance [2]. Anoikis is a special form of apoptosis, resulting from the loss of adhesion of cells to the extracellular matrix [3]. Anti-anoikis ability was first discovered in ovarian cancer by Frankel et al. [4]. Anoikis resistance acquired by ovarian cancer cells is essential for their survival in ascites and is the first step in achieving distant dissemination [5]. Moreover, ovarian cancer cells are more resistant to chemotherapy when grown in the form of multicellular spheroids than in monolayers [6]. Although the existence of anoikis is well recognized, little is known about the mechanisms of the regulation of anoikis, especially the crosstalk between anoikis and other clinically important cellular events.

Epithelial-mesenchymal transition (EMT) is an essential event during the acquisition of anoikis resistance by epithelial-derived cells. A mesenchymal phenotype usually confers the anoikis resistance to tumor cells $[7,8]$. Thus, there may be common regulators involved in the acquisition of anoikis and mesenchymal phenotype [9]. However, the role of known EMT regulators in anoikis needs to be elucidated experimentally. Most of the sequences in the human genome are transcribed into non-protein-coding RNA molecules, among which RNA transcripts longer than 200 nucleotides are called long non-coding RNAs (lncRNAs) [10]. LncRNAs are important regulators of transcription and translation and participate in many biological processes such as cell proliferation, apoptosis, and differentiation. These universal functions 
of lncRNAs suggest their involvement in tumorigenesis [11]. The lncRNA, HOX transcript antisense RNA (HOTAIR), has been shown to be an indicator of poor prognosis in ovarian cancer [12]. Overexpression of HOTAIR enhances invasion, migration, and tolerance to cisplatin in ovarian cancer cells, via a mechanism related to EMT [13-16]. Therefore, in this study, we investigated the role of HOTAIR in the acquisition of the anoikis resistance in ovarian cancer cells as well as its underlying molecular mechanism.

\section{Materials and methods}

Cell line and culture method. The ovarian cancer cell line, SKOV3 that has an intermediate mesenchymal phenotype with high spheroidogenic efficiency [17], was purchased from the Shanghai Institute of Biochemistry and Cell Biology (Shanghai, China) and used in this study. Cells were maintained in complete high-glucose Dulbecco's modified Eagle's medium (DMEM) supplemented with $10 \%$ fetal bovine serum (FBS) and 1\% penicillin and streptomycin. All the supplements used in the culture medium were purchased from HyClone (Logan, UT, USA). The cells were cultured at $37^{\circ} \mathrm{C}$ in a humidified atmosphere containing $5 \% \mathrm{CO}_{2}$.

Induction of anoikis. The anoikis induction method used in this study was adapted from a previous report [18]. Adherent cells were dissociated using trypsin and strained through a $40 \mu \mathrm{m}$ pore strainer (BD Biosciences, Franklin Lakes, NJ, USA) to obtain a single-cell suspension. The cell suspension was seeded at a density of $1 \times 10^{5}$ cells/well in the ultra-low attachment (ULA) 6-well plates (Corning Inc., Corning, NY, USA). The cells were cultured overnight in serum-free DMEM and strained as described above to remove cell aggregates to avoid false-positive results. For the induction of anoikis, the cells were seeded back in ULA plates and cultured further for 24,48 , or $72 \mathrm{~h}$ prior to the assessment of their morphology and flow cytometric analysis. For assessment of continuous spheroid formation, cells were cultured further in ULA plates for a period of 2 weeks and

Table 1. The primers used in real-time PCR.

\begin{tabular}{ll}
\hline Gene & Primer sequence (5'-3') \\
\hline HOTAIR & F: ACAGTGGCACCGCTTTTCTA \\
& R: GCAGGGTCCCACTGCATAAT \\
miR-193a & RT: GTCGTATCCAGTGCAGGGTCCGAGGTATT \\
& CGCACTGGATACGACATGGG \\
& F: CGAACTGGCCTACAAAGTC \\
& R: GTGCAGGGTCCGAGGT \\
DOK2 & F: ACCAGTGTCGTTTAAGGAAC \\
& R: GACCTCTCCCGTTGAAACTC \\
U6 & RT: AAAATATGGAACGCTTCACGAATTTG \\
& F: CTCGCTTCGGCAGCACATATACT \\
& R: ACGCTTCACGAATTTGCGTGTC \\
GAPDH & F: GAAGGTCGGAGTCAACGGATTT \\
& R: CCTGGAAGATGGTGATGGGATT \\
\hline
\end{tabular}

the number of spheres was counted under a microscope. The spheres were then collected, dispersed, seeded into ULA plates $\left(1 \times 10^{5}\right.$ cells/well), and the culture process was then repeated for two passages.

Detection of apoptosis. After induction of anoikis, the collected cells were dispersed by trypsinization and analyzed by flow cytometry using APC Annexin V/propidium iodide (PI) Apoptosis Detection Kit (BioLegend, San Diego, CA, USA). The cells were washed with cold phosphate-buffered saline (PBS), suspended in annexin $\mathrm{V}$ binding buffer (BioLegend), and adjusted to a density of $1 \times 10^{5}$ cells $/ \mathrm{ml}$. The cells were then stained with annexin V/PI detection reagents for $30 \mathrm{~min}$ in the dark. Cell apoptosis was detected by FACSCalibur flow cytometry (BD).

RNA extraction and qRT-PCR. Total RNA was extracted from the collected cells using TRIzol reagent (Invitrogen, Carlsbad, CA, USA). Single-strand cDNA was reverse transcribed from the RNA template using the SuperScript III First-Strand Synthesis System (Invitrogen). Expression of selected genes was detected using quantitative RT-PCR on an ABI 7500 Real-Time PCR System (Applied Biosystems, Bedford, MA, USA) with specific primers (Table 1). The primers were synthesized by GenePharma (Shanghai, China). AceQ qPCR SYBR Green Master mix (with ROX) was purchased from Vazyme Biotech Co. Ltd. (Nanjing, China) and used in the PCR reactions. The PCR conditions used were as follows: denaturation at $95^{\circ} \mathrm{C}$ for $10 \mathrm{~min}$, followed by 40 amplification cycles of $95^{\circ} \mathrm{C}$ for $10 \mathrm{~s}, 60^{\circ} \mathrm{C}$ for $30 \mathrm{~s}$, and $72^{\circ} \mathrm{C}$ for $10 \mathrm{~s}$. The relative mRNA amount of target transcripts was normalized to the amount of U6 or GAPDH in the same sample using the $\Delta \Delta \mathrm{Ct}$ method [19].

Protein extraction and western blot analysis. Total protein was extracted from approximately $5 \times 10^{6}$ cells by lysing the cells in Total Protein Extraction Reagent for cell cultures (Boster Biological Technology, Wuhan, China). Protein concentration was measured using a BCA Protein Assay Kit (Boster). For each sample, $50 \mu \mathrm{g}$ of total protein was separated by $12 \%$ sodium dodecyl sulfate-polyacrylamide gel electrophoresis and then transferred onto a polyvinylidene difluoride (PVDF) membrane (Millipore, Billerica, MA, USA). After blocking with 5\% non-fat milk, immunoblotting was performed by incubating the membranes overnight at $4{ }^{\circ} \mathrm{C}$ with the following primary antibodies: rabbit monoclonal anti-N-cadherin (1:1000, M01577-2), rabbit monoclonal anti-ZEB1 (1:500, BM5462), rabbit polyclonal anti-TWIST (1:500, A12525), rabbit monoclonal anti-Ecadherin (1:2000, BM4867), rabbit monoclonal anti-ErbB (1:1000, BM4149), rabbit monoclonal anti-EZH2 (1:300, BM4874), and mouse monoclonal anti-H3K27me3 (1:1000, ab6002, Abcam Inc., Cambridge, UK). The membranes were then incubated with appropriate horseradish peroxidaseconjugated goat anti-rabbit IgG (BA1055) or goat antimouse IgG (BA1051, both 1:2000) for $1 \mathrm{~h}$. All the antibodies were purchased from Boster unless indicated. Protein bands were visualized using an Enhanced Chemiluminescence Kit 
(Boster). Relative levels of the target proteins were quantified using ImageJ software and normalized to that of GAPDH.

Small interfering RNA (siRNA) design and transfection. Specific siRNA sequence for inhibiting HOTAIR expression and a scrambled sequence were adapted from a previous report [20]. SKOV3 cells were seeded in 24-well plates at a density of $2 \times 10^{5}$ cells/well and grown overnight for adhesion. Double-stranded siRNA oligomers and scrambled sequences were then transfected into SKOV3 cells using Attractene Transfection Reagent (Qiagen, Hilden, Germany) following the manufacturer's instructions. After $48 \mathrm{~h}$ of transfection, cells were harvested and subjected to anoikis induction.

Wound healing assay. Following $72 \mathrm{~h}$ of anoikis induction, the spheroid cells were collected and dispersed into single-cell suspension. Cells were inoculated in 6-well plates $\left(1 \times 10^{6}\right.$ cells/well $)$ and cultured in complete DMEM to form a monolayer. A straight scratch line was made in the monolayer using a pipette tip. The cell debris was gently removed with PBS. The remaining adherent cells were cultured as a normal adherent culture. The scratch width was measured at 0 and $24 \mathrm{~h}$ after scratching under a microscope.

Matrigel transwell assay. Following $72 \mathrm{~h}$ of anoikis induction, the spheroid cells were harvested and adjusted to a density of $5 \times 10^{5}$ cells $/ \mathrm{ml}$ in serum-free DMEM. The cells were seeded in the upper insert of a matrigel-coated transwell plate (Millipore, Temecula, CA, USA) at a density of $1 \times 10^{5}$ cells/well and cultured overnight for adhesion. The next day, $1 \mathrm{ml}$ of complete DMEM was added to the lower chamber. After $48 \mathrm{~h}$ of culture, the cells remaining in the upper insert were removed along with the medium. The inserts were then fixed in 10\% formaldehyde for $30 \mathrm{~min}$ and stained with $0.1 \%$ crystal violet for $30 \mathrm{~min}$. The number of migrated cells was counted under a microscope. Five random fields were counted for each well.

Cell viability assay. Following $72 \mathrm{~h}$ of anoikis induction, the spheroid cells were collected and seeded in 96-well plates at a density of $2 \times 10^{4}$ cells/well and cultured overnight for adhesion. The cells were then treated with different concentrations $(0.4,0.8,1,2,4,8,10,15$, and $20 \mu \mathrm{M})$ of cisplatin (Sigma-Aldrich, St. Louis, MO, USA). Cells cultured without cisplatin were used as the normalization control. After incubation for $48 \mathrm{~h}$, cell viability was determined using an Enhanced Cell Counting Kit-8 (Beyotime Biotechnology, Shanghai, China) as the manufacturer's instructions. The optical density at $450 \mathrm{~nm}$ was measured using a microplate reader (Bio-Rad, Hercules, CA, USA). Percentage of cell viability was calculated as $\left(\mathrm{OD}_{\text {treatment }}-\mathrm{OD}_{\text {blank }}\right) /\left(\mathrm{OD}_{\text {normal }}-\right.$ $\left.\mathrm{OD}_{\text {blank }}\right)$.

Statistical analysis. Data were analyzed using GraphPad Prism 7 software. Continuous variables are expressed as mean \pm standard error of mean (SEM). Comparisons between two groups were performed using Student t test. Multiple comparisons were performed using one-way ANOVA followed by Tukey's post hoc analysis. The significance of all tests was set at $\mathrm{p}<0.05$.

\section{Results}

HOTAIR expression is upregulated in anoikis-resistant SKOV 3 cells. First, we induced anoikis in SKOV3 cells using a suspension culture system in ULA plates. The cells grew into large and loose clusters with a smooth surface in suspension culture (Figure 1A). As shown in Figure 1B, the HOTAIR expression level increased in the suspension cells in a time-dependent manner $(\mathrm{p}=0.0018)$, whereas its expression in the adherent culture cells did not change significantly $(p=0.9996)$. There was a significant difference in the HOTAIR expression trend between the two groups $(\mathrm{F}=25.53$, $\mathrm{p}=0.0003$ ). Following $72 \mathrm{~h}$ of suspension culture, the expression of HOTAIR in the suspension cells was significantly higher than that in the adherent cells $(\mathrm{p}<0.01)$. The apoptotic rate of both groups of cells increased with increased duration in culture but the rate of increase of the suspension cells was slower than that of the adherent cells $(\mathrm{F}=20.84, \mathrm{p}=0.0006)$. Moreover, the apoptotic rate of the suspension cells was significantly lower than that of the adherent cells after $72 \mathrm{~h}$ of suspension culture $(p<0.01)$. These results suggest that SKOV3 cells attain anoikis resistance following the suspension culture. Moreover, this resistance is related to the upregulation of HOTAIR expression levels.

HOTAIR increased anoikis resistance and spheroid formation of SKOV 3 cells. To further determine the effect of HOTAIR expression on anoikis resistance, we inhibited the HOTAIR expression in SKOV3 cells using siRNA technology (Figure 2A). We then evaluated the cellular apoptotic rate of the cells after $72 \mathrm{~h}$ of suspension culture. The apoptotic rate of the si-HOTAIR group increased 3-fold more than that of the non-interference control group $(\mathrm{p}<0.01$; Figure $2 \mathrm{~B})$. These results suggest that downregulation of the HOTAIR expression counteracts the effect of suspension culture and increases the apoptosis of cells growing in suspension. Continuous spheroid forming ability is an important indicator of selfrenewal ability. As shown in Figure 2C, compared to primary cultures, the spheroid forming ability of the third passage cells was enhanced in all groups (both $\mathrm{p}<0.01$ ). However, the spheroid formatting ability was significantly reduced in the si-HOTAIR group compared to the control group $(\mathrm{p}<0.05)$. The results further suggest that the HOTAIR expression is associated with the regeneration ability of SKOV3 cells after the loss of adhesion, and decreased levels of HOTAIR reduce the ability to develop anoikis resistance.

HOTAIR is associated with the aggressiveness of spheroid cells. The acquisition of anoikis resistance is a prerequisite for ovarian cancer cells to survive in ascites and develop distant metastasis and is related to drug resistance $[5,6]$. Next, we investigated the effect of HOTAIR levels on the malignant characteristics of the spheroid cells. As shown in Figure 3A, inhibiting HOTAIR expression limited cellular migration ability. Compared to the cells in the control group, fewer cells in the si-HOTAIR group migrated towards the scratch $(\mathrm{p}<0.01)$. In addition, the invasiveness of spheroid 
A

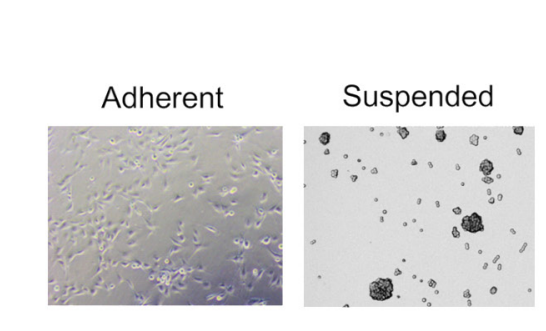

B

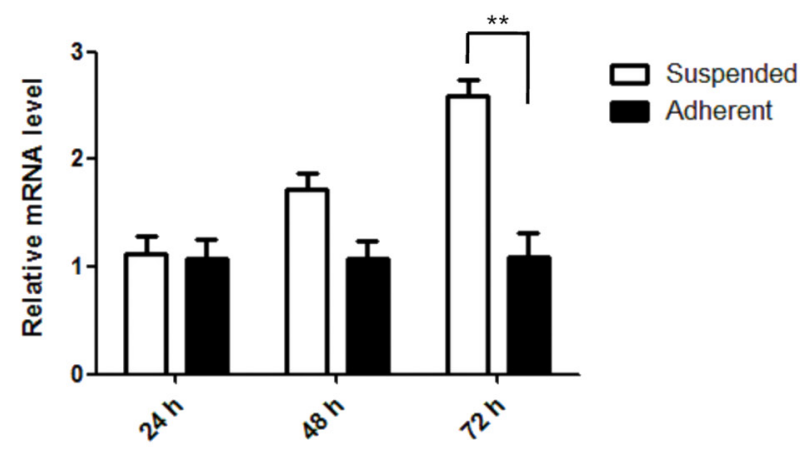

C
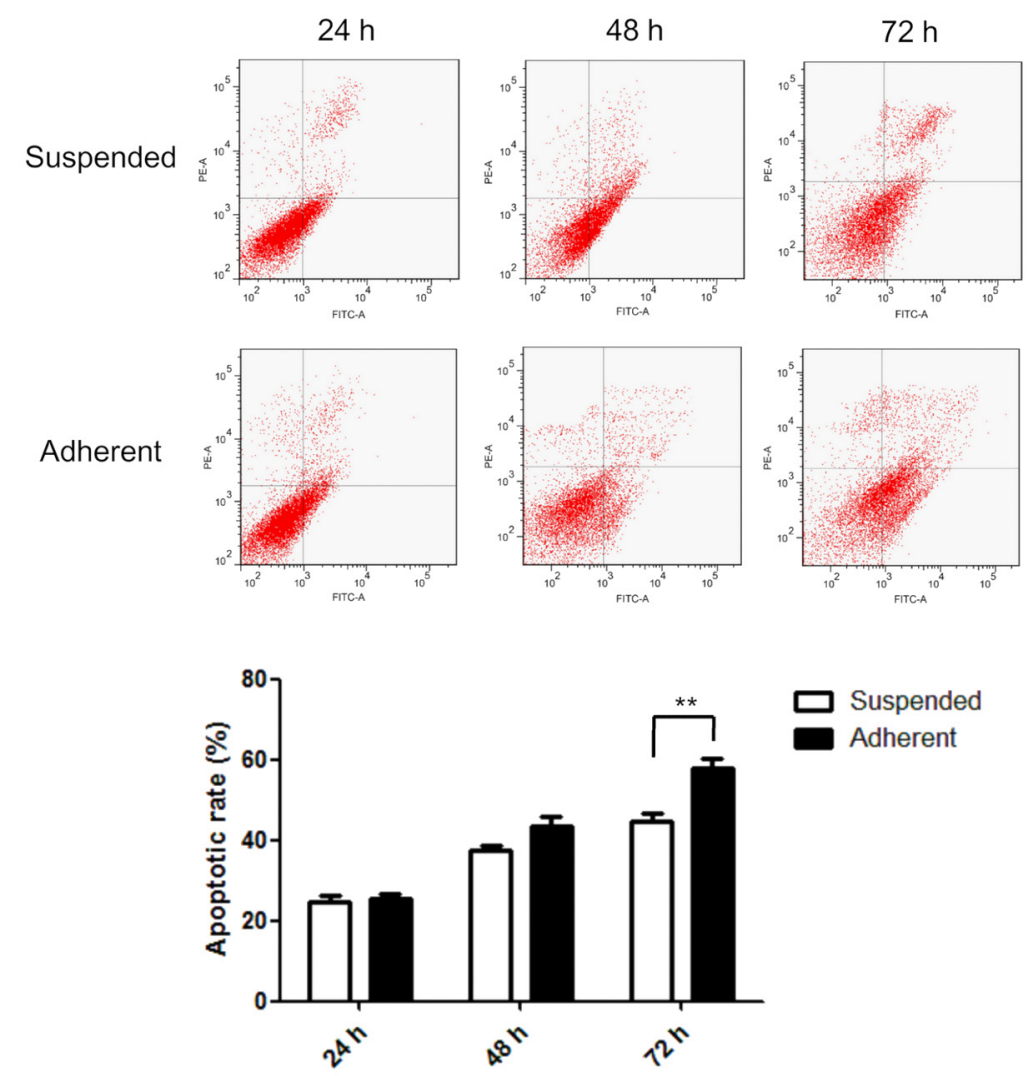

Figure 1. HOTAIR expression is upregulated in anoikis resistant SKOV3 cells. A) Image of typical spheroids. Scale bar, $100 \mu \mathrm{m}$. B) Relative HOTAIR expression in suspension and adherent cells. C) Cellular apoptosis (\%) in suspension and adherent cells. Error bars represent SEM from triplicate experiments. ${ }^{* *} \mathrm{p}<0.01$.

cells also decreased following the inhibition of HOTAIR expression. Compared to the cells in the control group, fewer cells in the si-HOTAIR group invaded the matrigel layer $(p<0.01$, Figure $3 B)$. These results suggest that the suspension culture cells have a weakened ability to degrade the extracellular matrix and migrate to form new lesions following inhibition of HOTAIR expression. Next, the chemoresistance of the spheroid cells was evaluated. After $48 \mathrm{~h}$ of cisplatin treatment, cell viability of the si-HOTAIR group was significantly lower than that of the control group ( $\mathrm{F}=1510$, $\mathrm{p}<0.01$, Figure $3 \mathrm{C}$ ). The half-maximal inhibitory concentration $\left(\mathrm{IC}_{50}\right.$ ) of cisplatin in the si-HOTAIR group was $0.901 \mu \mathrm{M}$ (95\% confidence intervals [CI]: 0.834-0.958 $\mu \mathrm{M}$ ), which was lower than that in the control group $(1.829 \mu \mathrm{M}, 95 \%$ CI: $1.558-2.100 \mu \mathrm{M})$. We then examined the protein expression of representative EMT factors in different spheroid cells. As shown in Figure 3D, the epithelial phenotype-related molecules, N-cadherin, ZEB1, and TWIST, were downregulated in the si-HOTAIR group, whereas the mesenchymal phenotype-related molecules, E-cadherin and ErbB, were 
upregulated in the si-HOTAIR group. These results suggest that the expression level of HOTAIR is related to the migration, invasion, and chemoresistance of spheroid cells. During this process, the protein expression profile is more inclined to reflect the characteristics of the epithelial phenotype.

EZH2 recruitment mediates the acquisition of anoikis resistance. An important regulatory mechanism of HOTAIR is to induce the trimethylation of histone $\mathrm{H} 3$ lysine 27 (H3K27me3) by recruiting EZH2 [21]. H3K27me3 transcriptionally silences tumor suppressor genes and promotes tumor growth and metastasis [22]. Therefore, we then analyzed whether EZH2 recruitment and H3K27me3 were involved in the acquisition of anoikis resistance associated with HOTAIR expression. First, we compared the expression of EZH2 and $\mathrm{H} 3 \mathrm{~K} 27 \mathrm{me} 3$ before and after anoikis-induction. As shown in Figure 4A, the expression of both EZH2 and $\mathrm{H} 3 \mathrm{~K} 27 \mathrm{me} 3$ was significantly increased in the suspension cells after $72 \mathrm{~h}$ of anoikis induction (both $\mathrm{p}<0.01$ ), but remained unaltered in the adherent cells. Following inhibition of HOTAIR expression in the spheroid cells, the expression of both EZH2 and H3K27me3 decreased significantly $(\mathrm{p}<0.05$ and $\mathrm{p}<0.01$ vs. control, respectively; Figure $4 \mathrm{~B}$ ). A previous study reported that miR-193a and DOK2 are signature tumor suppressor genes that are regulated via promoter methylation [23]. Consistently, in our study, the expression of miR-193a and DOK2 was upregulated in the spheroid cells in the si-HOTAIR group $(\mathrm{p}<0.05$ and $\mathrm{p}<0.01$ vs. control, respectively, Figure 4C). These results suggest that the regulation of $\mathrm{EZH} 2$-mediated methylation constitutes one possible mechanism through which HOTAIR regulates anoikis resistance-related biological characteristics.

\section{Discussion}

HOTAIR was first reported by Rinn et al. as a trans-acting transcriptional repressor of the HOXD locus [24]. Several studies have shown that HOTAIR is an oncogenic lncRNA differentially expressed in ovarian cancer tissues $[12,15$, 16]. Our study showed that HOTAIR is upregulated in ovarian cancer cells in suspension culture and enables the suspension cells to acquire anoikis resistance. Silencing of HOTAIR in SKOV3 cells inhibited spheroid formation ability, reduced aggressiveness, and enhanced chemosensitivity. The classical epigenetic regulatory mechanism of HOTAIR participated in this process.

Our study suggests that EZH2-mediated H3K27me3 is associated with anoikis induction
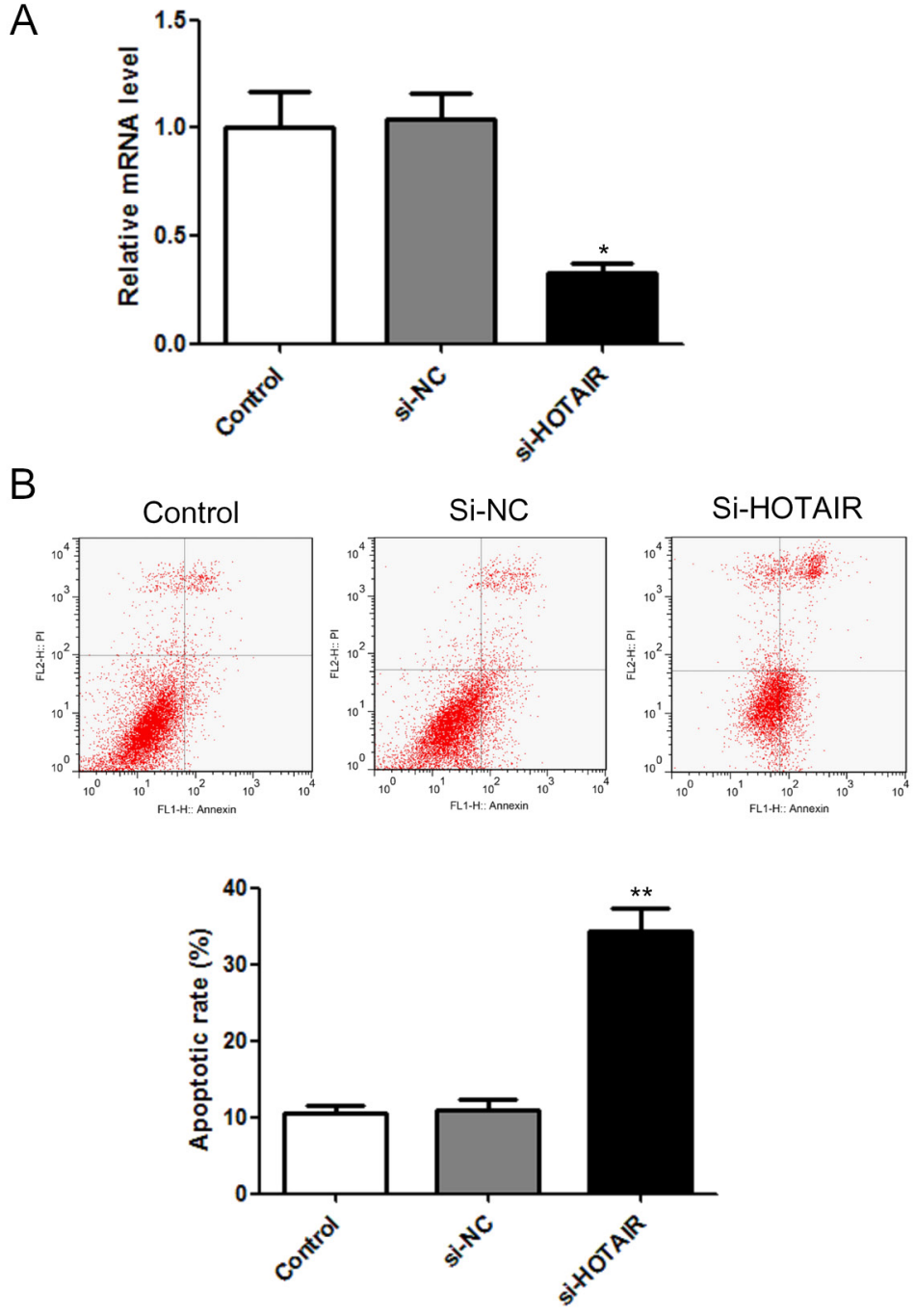

C

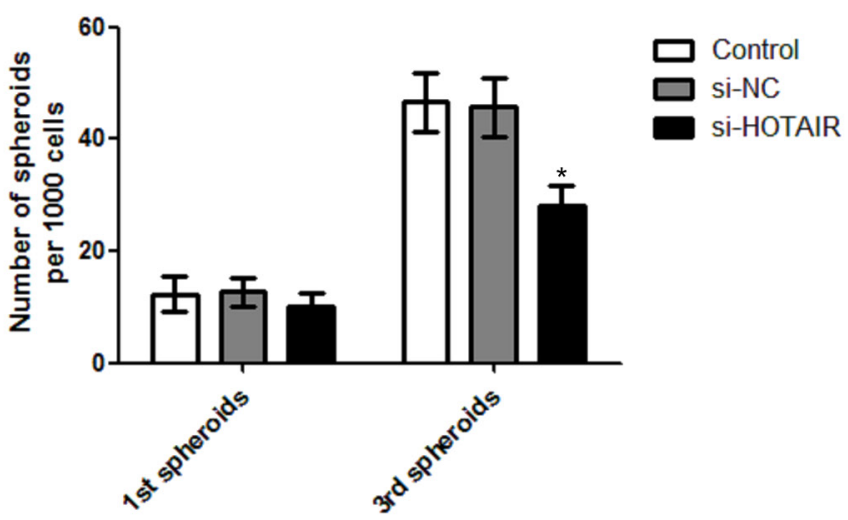

Figure 2. HOTAIR increased anoikis resistance and spheroid forming ability of SKOV3 cells. A) Relative HOTAIR expression in cells transfected with si-HOTAIR or si-NC (scrambled). Non-transfected cells were used as a control. B) Spheroid forming ability of different cells in primary and tertiary cultures. C) Cellular apoptosis (\%) in different cells. Error bars represent SEM from triplicate experiments. ${ }^{*} \mathrm{p}<0.05$ vs. control; ${ }^{* *} \mathrm{p}<0.01$ vs. control 

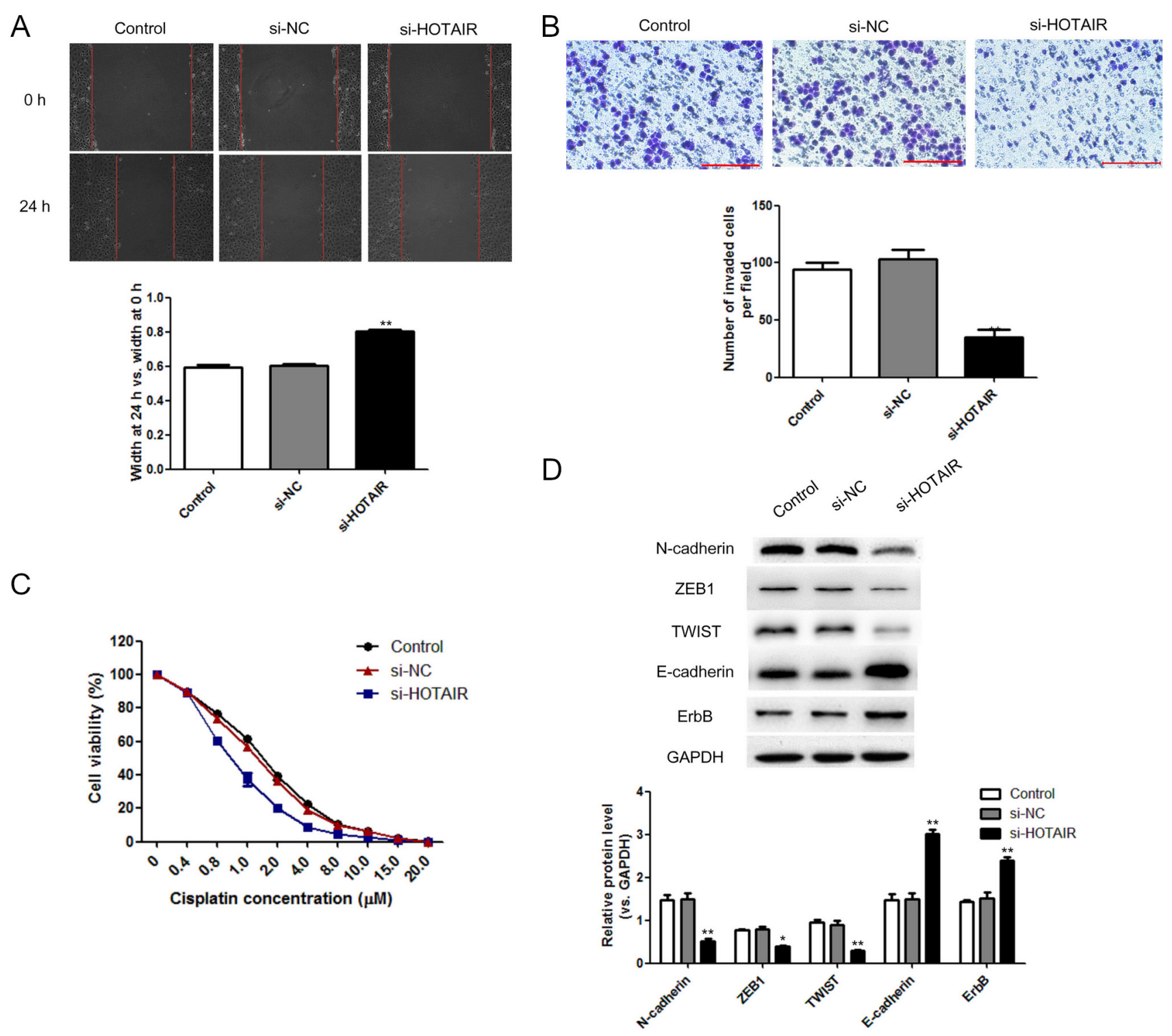

Figure 3. HOTAIR expression is associated with the aggressiveness of spheroid cells. A) Migration ability of cells transfected with si-HOTAIR or si-NC. Non-transfected cells were used as a control. B) Invasion ability of different cells. C) Cell viability following treatment with different concentrations of cisplatin. D) Relative protein expression of representative EMT factors. Error bars represent SEM from triplicate experiments. ${ }^{*} \mathrm{p}<0.05 \mathrm{vs.}$ control; ${ }^{* *} \mathbf{p}<\mathbf{0 . 0 1}$ vs. control

that is relevant to EMT. Similarly, a previous study reported that EZH2 controls EMT and anoikis in colon cancer [25], suggesting a connection between epigenetic regulation and EMT. Our study clarifies the molecular mechanism by which EZH2 expression and corresponding EMT-related events are regulated by HOTAIR. Heat shock protein 90 (Hsp90) is another joint regulator of EZH2 and EMT in prostate cancer cells [26]. These studies indicate that epigenetic regulation and EMT are relevant events. Therefore, targeting their common triggers may be a potential strategy to improve treatment outcomes in such cancers.
A recent study has shown that activation of phosphatidylinositol 3 kinase (PI3K) and AKT pathway is a key event in the acquisition of anoikis resistance [4]. EZH2 is also regulated by the PI3K/AKT pathway [25, 27]. HOTAIR can work as competing endogenous RNA (ceRNA) to activate the expression of PI3K regulatory subunit 3 (PIK3R3), thus promoting the malignant characteristics of ovarian cancer cells [28]. Therefore, the PI3K pathway may be the signaling pathway that mediates the regulation of EZH2 by HOTAIR. In addition, activation of the PI3K-AKT pathway is associated with enhanced expression of Bcl- $\mathrm{X}_{\mathrm{L}}, \mathrm{RAB} 25$, 
A

$\frac{\text { Suspended }}{0 \mathrm{~h} 72 \mathrm{~h}} \frac{\text { Adherent }}{0 \mathrm{~h} 72 \mathrm{~h}}$

$\mathrm{EZH} 2$

H3K27me3

GAPDH

$\mathrm{EZH} 2$

H3K27me3
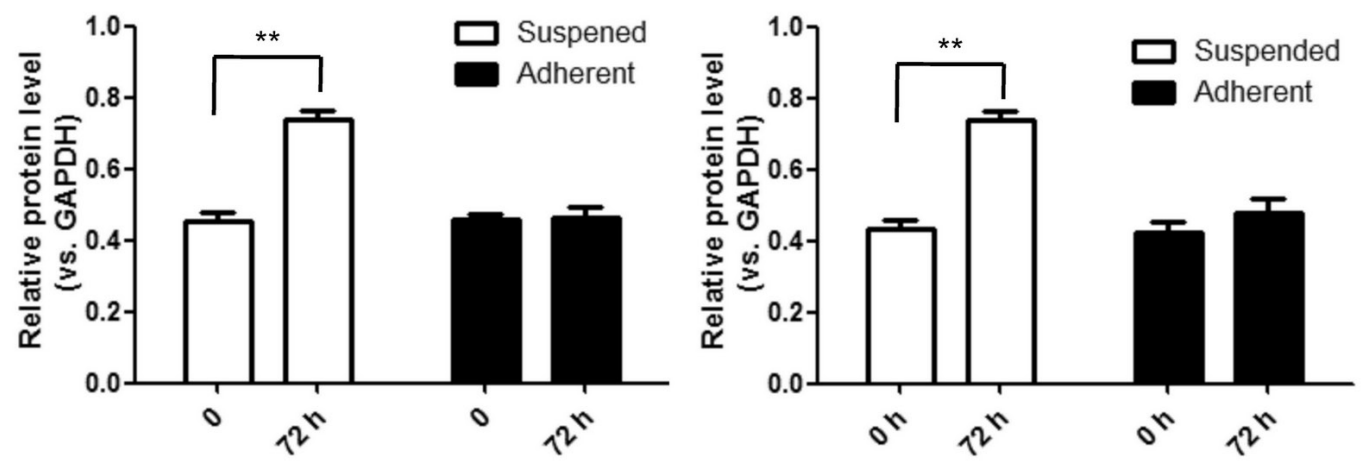

B

C

miR-193a
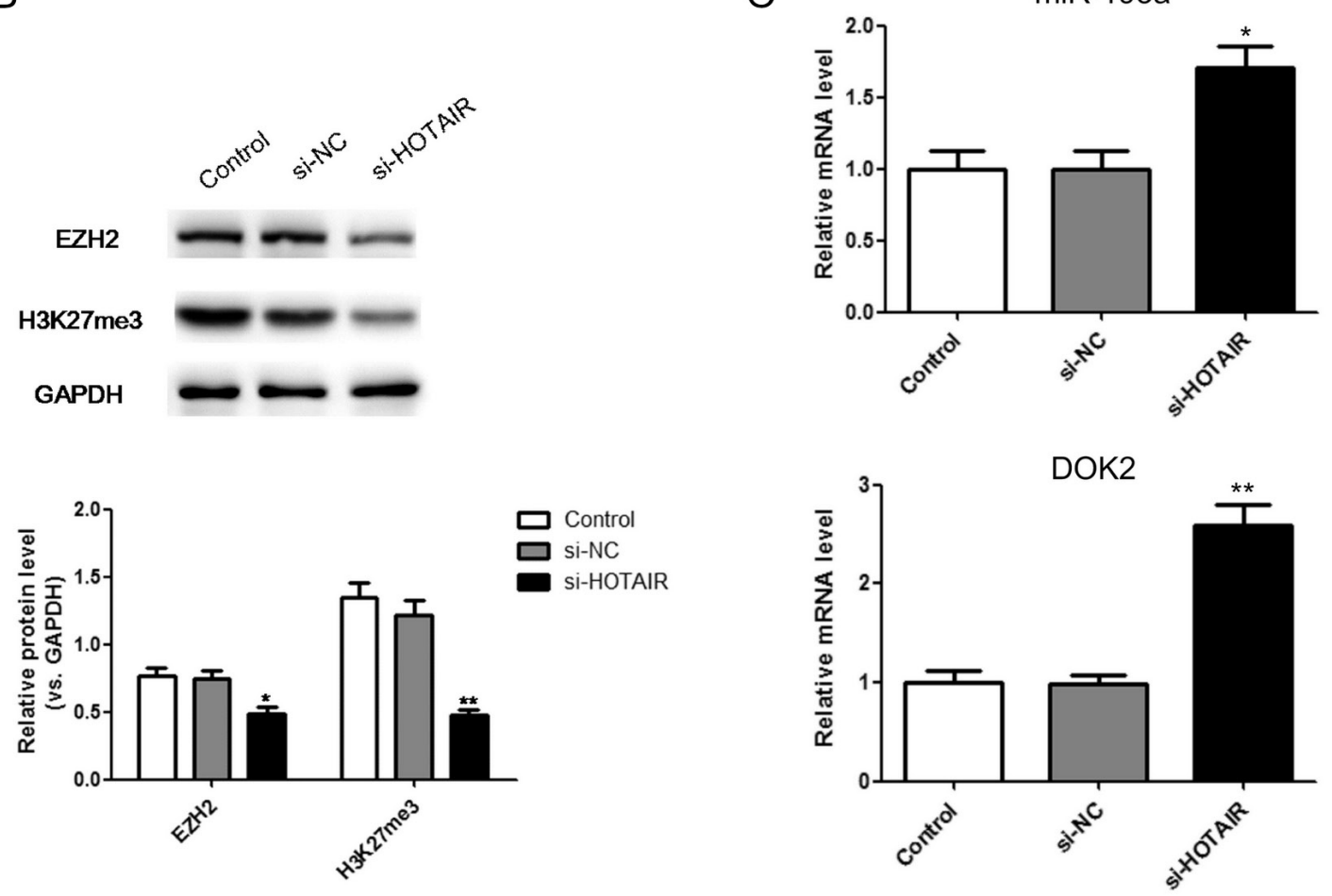

Figure 4. Recruitment of EZH2 mediates the acquisition of anoikis resistance related to HOTAIR expression. A) Protein expression of EZH2 and H2K27me3 in suspension and adherent cells. ${ }^{* *}$ p $<0.01$. B) Protein expression of EZH2 and H2K27me3 in cells transfected with si-HOTAIR or si-NC (scramble). Non-transfected cells were used as a control. C) Relative mRNA expression of miR-193a and DOK2, representative target genes regulated by methylation. Error bars represent SEM from triplicate experiments. ${ }^{\star} \mathrm{p}<0.05$ vs. control; ${ }^{* *}$ p $<0.01$ vs. control 
and tyrosine kinase receptor B (TrkB) [29-31]. Expression of c-Met is required for anoikis resistance and its effect depends on the crosstalk between the extracellular signal-regulated kinase (ERK) $1 / 2$ and PI3K pathways [18]. HtrA1 is another regulator of the AKT pathway and contributes to metastatic suppression by promoting anoikis in ovarian cancer [32]. The endocrine system also affects the level of anoikis. In human ovarian cancer patients, enhanced adrenergic activity is associated with increased phosphorylation of focal adhesion kinase Y297 and lower levels of anoikis [33].

One of the main findings of this study is that HOTAIR expression level is related to anoikis resistance acquired by ovarian cancer cells. Previous studies have revealed several mechanisms underlying the oncogenic role of HOTAIR. HOTAIR promotes the expression of nuclear factor kappa-B and interleukin 6 , thereby regulating tumor cell aging and chemoresistance [14]. By regulating the expression of matrix metalloproteinases and EMT-related genes, HOTAIR promotes lymph node metastasis and shortens overall survival and disease-free survival in patients with epithelial ovarian cancer [16]. HOTAIR also promotes ovarian cancer progression by sponging microRNA (miRNA)-1, -214-3p, $-330-5 p,-214,-217,-206$, and $-373[28,34-36]$. Our study highlights a potentially new mechanistic role for HOTAIR in ovarian cancer, that is to improve the tolerance of tumor cells to apoptosis due to inadequate cell-matrix interaction. Similar results were reported in a study of glioma. The lncRNA highly upregulated in liver cancer (HULC) plays an oncogenic role and silencing HULC-induced anoikis through the PI3K/AKT/mTOR pathway [37]. miR-26a was reported to suppress hepatocellular carcinoma through inducing anoikis [38]. Currently, there are limited reports on the role of non-coding RNAs in anoikis and further research is needed on this topic.

In our study, we only studied the effect of inhibition of HOTAIR, mainly because HOTAIR is overexpressed in ovarian cancer cells. In addition, inhibiting HOTAIR expression using siRNA is technically easier for us to implement. Most of the related researches of HOTAIR also adopts the same research strategy by interfering with HOTAIR expression. Although we did not study the effect of overexpressing HOTAIR on the anoikis, its effect on other cellular phenotypes can be found in the literature. Zhang et al. reported that upregulation of HOTAIR enhanced cell viability in HeyC2 ovarian cancer cells [35]. Li et al. reported that overexpression of HOTAIR promoted cell viability and inhibited apoptosis in cervical cancer cells (HeLa and C33A) [39]. It can be inferred from these studies that overexpression of HOTAIR may enhance the malignancy of tumor cells.

The ability to escape anoikis is essential for ovarian cancer cells because cancer progression depends on the spread of tumor cells along the peritoneum and malignant ascites. Therefore, promoting anoikis may be an optimal choice for the treatment of ovarian cancer. There have been a few pilot studies in this area. Foretinib, a small-molecule inhibitor of
c-Met, suppresses the growth of xenograft tumors in nude mice through the induction of anoikis [40]. Considering the importance of the PI3K pathway activation in anoikis, PI3K inhibitors may be expected to suppress tumorigenesis by increasing anoikis. However, the development of PI3K targeted drugs has been a challenge. Recently, the first drug, aplelisib, was approved for the treatment of breast cancer [41]. Other candidate drugs are still in the preclinical or clinical trial stage, but the prospect is worth looking forward to. In summary, although the mechanism of tumor anoikis remains to be clarified, it is a promising field for the development of new treatment strategies. Our study provides new insights into the crosstalk between anoikis and other key biological mechanisms, as well as the identification of common regulatory factors.

Acknowledgments: We would like to thank Editage (www.editage.com) for English language editing.

\section{References}

[1] LHEUREUX S, GOURLEY C, VERGOTE I, OZA AM. Epithelial ovarian cancer. Lancet 2019; 393: 1240-1253. https:// doi.org//10.1016/S0140-6736(18)32552-2

[2] MATSUO K, LIN YG, ROMAN LD, SOOD AK. Overcoming platinum resistance in ovarian carcinoma. Expert Opin Investig Drugs 2010; 19: 1339-1354. https://doi.org//10.151 7/13543784.2010.515585

[3] SIMPSON CD, ANYIWE K, SCHIMMER AD. Anoikis resistance and tumor metastasis. Cancer Lett 2008; 272: 177185. https://doi.org//10.1016/j.canlet.2008.05.029

[4] FRANKEL A, ROSEN K, FILMUS J, KERBEL R. Induction of anoikis and suppression of human ovarian tumor growth in vivo by down-regulation of $\mathrm{Bcl}-\mathrm{X}(\mathrm{L})$. Cancer Res 2001; 61: 4837-4841.

[5] KIM Y, KOO KH, SUNG JY, YUN U, KIM H. Anoikis resistance: an essential prerequisite for tumor metastasis. Int J Cell Biol 2012; 2012: 306879. https://doi. org//10.1155/2012/306879

[6] LIAO J, QIAN F, TCHABO N, MHAWECH-FAUCEGLIA P, BECK A et al. Ovarian cancer spheroid cells with stem cell-like properties contribute to tumor generation, metastasis and chemotherapy resistance through hypoxia-resistant metabolism. PLoS One 2014; 9: e84941. https://doi. org//10.1371/journal.pone.0084941

[7] MANI SA, GUO W, LIAO MJ, EATON EN, AYYAKKANNU A et al. The epithelial-mesenchymal transition generates cells with properties of stem cells. Cell 2008; 133: 704-715. https://doi.org//10.1016/j.cell.2008.03.027

[8] GUADAMILLAS MC, CEREZO A, POZO MA. Overcoming anoikis--pathways to anchorage-independent growth in cancer. J Cell Sci 2011; 124: 3189-3197. https://doi.org//10.1242/ jcs.072165

[9] FRISCH SM, SCHALLER MD, CIEPLY B. Mechanisms that link the oncogenic epithelial-mesenchymal transition to suppression of anoikis. J Cell Sci 2013; 126: 21-29. https://doi. org//10.1242/jcs. 120907 
[10] CAO J. The functional role of long non-coding RNAs and epigenetics. Biol Proced Online 2014; 16: 42. https://doi. org//10.1186/1480-9222-16-11

[11] GIBB EA, BROWN CJ, LAM WL. The functional role of long non-coding RNA in human carcinomas. Mol Cancer 2011; 10: 38. https://doi.org//10.1186/1476-4598-10-38

[12] LI J, WEN W, ZHAO S, WANG J, CHEN J et al. Prognostic role of HOTAIR in four estrogen-dependent malignant tumors: a meta-analysis. Onco Targets Ther 2015; 8: 14711482. https://doi.org//10.2147/OTT.S84687

[13] TESCHENDORFF AE, LEE S, JONES A, FIEGL H, KALWA $\mathrm{M}$ et al. HOTAIR and its surrogate DNA methylation signature indicate carboplatin resistance in ovarian cancer. Genome Med 2015; 7: 108. https://doi.org//10.1186/s13073015-0233-4

[14] OZES AR, MILLER DFB, OZES ON, FANG F, LIU Y et al. NF- $\mathrm{B}$-HOTAIR axis links DNA damage response, chemoresistance and cellular senescence in ovarian cancer. Oncogene 2016; 35: 5350-5361. https://doi.org//10.1038/onc.2016.75

[15] WANG Y, WANG H, SONG T, ZOU Y, JIANG J et al. HOTAIR is a potential target for the treatment of cisplatin-resistant ovarian cancer. Mol Med Rep 2015; 12: 2211-2216. https://doi.org//10.3892/mmr.2015.3562

[16] QIU J, LIN Y, YE L, DING J, FENG W et al. Overexpression of long non-coding RNA HOTAIR predicts poor patient prognosis and promotes tumor metastasis in epithelial ovarian cancer. Gynecol Oncol 2014; 134: 121-128. https://doi. org//10.1016/j.ygyno.2014.03.556

[17] HUANG RY, WONG MK, TAN TZ, KUAY KT, NG A et al. An EMT spectrum defines an anoikis-resistant and spheroidogenic intermediate mesenchymal state that is sensitive to e-cadherin restoration by a src-kinase inhibitor, saracatinib (AZD0530). Cell Death Dis 2013; 4: e915. https:// doi.org//10.1038/cddis.2013.442

[18] TANG MKS, ZHOU HY, YAM JWP, WONG AST. c-Met overexpression contributes to the acquired apoptotic resistance of nonadherent ovarian cancer cells through a cross talk mediated by phosphatidylinositol 3-kinase and extracellular signal-regulated kinase 1/2. Neoplasia 2010; 12: 128138. https://doi.org//10.1593/neo.91438

[19] LIVAK KJ, SCHMITTGEN TD. Analysis of relative gene expression data using real-time quantitative PCR and the 2(-Delta Delta C(T)) Method. Methods 2001; 25: 402-408. https://doi.org//10.1006/meth.2001.1262

[20] WANG J, CHEN D, HE X, ZHANG Y, SHI F et al. Downregulated lincRNA HOTAIR expression in ovarian cancer stem cells decreases its tumorgeniesis and metastasis by inhibiting epithelial-mesenchymal transition. Cancer Cell Int 2015; 15: 24. https://doi.org//10.1186/s12935-015-0174-4

[21] YOO KH, HENNINGHAUSEN L. EZH2 methyltransferase and H3K27 methylation in breast cancer. Int J Biol Sci 2012; 8: 59-65. https://doi.org//10.7150/ijbs.8.59

[22] BETANCUR JG, TOMARI Y. Cryptic RNA-binding by PRC2 components EZH2 and SUZ12. RNA Biol 2015; 12: 959-965. https://doi.org//10.1080/15476286.2015.1069463
[23] FANG F, CARDENAS H, HUANG H, JIANG G, PERKINS $\mathrm{SM}$ et al. Genomic and epigenomic signatures in ovarian cancer associated with resensitization to platinum drugs. Cancer Res 2017; 78: 631-644. https://doi.org//10.1158/0008-5472. CAN-17-1492

[24] RINN JL, KERTESZ M, WANG JK, SQUAZZO SL, XU X et al. Functional demarcation of active and silent chromatin domains in human HOX loci by noncoding RNAs. Cell 2007; 129: 1311-1323. https://doi.org//10.1016/j.cell.2007.05.022

[25] FERRARO A, MOURTZOUKOU D, KOSMIDOU V, AVLONITIS S, KONTOGEORGOS G et al. EZH2 is regulated by ERK/AKT and targets integrin alpha2 gene to control Epithelial-Mesenchymal Transition and anoikis in colon cancer cells. Int J Biochem Cell Biol 2013; 45: 243-254. https://doi. org//10.1016/j.biocel.2012.10.009

[26] NOLAN KD, FRANCO OR, HANCE MW, HAYWARD SW, ISAACS JS. Tumor-secreted Hsp90 subverts polycomb function to drive prostate tumor growth and invasion. J Biol Chem 2015; 290: 8271-8282. https://doi.org//10.1074/jbc. M115.637496

[27] RIQUELME E, BEHRENS C, LIN HY, SIMON G, PAPADIMITRAKOPOULOU V et al. Modulation of EZH2 expression by MEK-ERK or PI3K-AKT signaling in lung cancer is dictated by different KRAS oncogene mutations. Cancer Res 2016; 76: 675-685. https://doi.org//10.1158/0008-5472. CAN-15-1141

[28] DONG L, HUI L. HOTAIR promotes proliferation, migration, and invasion of ovarian cancer SKOV3 cells through regulating PIK3R3. Med Sci Monit 2016; 22: 325-331. https://doi.org//10.12659/msm.894913

[29] CHENG KW, LAHAD J, KUO WL, LAPUK A, YAMADA K et al. The RAB25 small GTPase determines aggressiveness of ovarian and breast cancers. Nat Med 2004; 10: 1251-1256. https://doi.org//10.1038/nm1125

[30] DODIER P, PICHE A. Bcl-X(L) is functionally non-equivalent for the regulation of growth and survival in human ovarian cancer cells. Gynecol Oncol 2006; 100: 254-263. https:// doi.org//10.1016/j.ygyno.2005.08.028

[31] YU X, LIU L, CAI B, HE Y, WAN X. Suppression of anoikis by the neurotrophic receptor TrkB in human ovarian cancer. Cancer Sci 2008; 99: 543-552. https://doi.org//10.1111/ j.1349-7006.2007.00722.x

[32] HE X, OTA T, LIU P, SU C, CHIEN J et al. Downregulation of HtrA1 promotes resistance to anoikis and peritoneal dissemination of ovarian cancer cells. Cancer Res 2010; 70: 3109-3118. https://doi.org//10.1158/0008-5472.CAN-093557

[33] SOOD AK, ARMAIZ-PENA GN, HALDER J, NICK AM, STONE RL et al. Adrenergic modulation of focal adhesion kinase protects human ovarian cancer cells from anoikis. J Clin Invest 2010; 120: 1515-1523. https://doi.org//10.1172/ JCI40802

[34] YIWEI T, HUA H, HUI G, MAO M, XIANG L. HOTAIR interacting with MAPK1 regulates ovarian cancer skov3 cell proliferation, migration, and invasion. Med Sci Monit 2015; 21: 1856-1863. https://doi.org//10.12659/MSM.893528 
[35] ZHANG Z, CHENG J, WU Y, QIU J, SUN Y et al. LncRNA HOTAIR controls the expression of Rab22a by sponging miR-373 in ovarian cancer. Mol Med Rep 2016; 14: 24652472. https://doi.org//10.3892/mmr.2016.5572

[36] CHANG L, GUO R, YUAN Z, SHI H, ZHANG D. LncRNA HOTAIR regulates CCND1 and CCND2 expression by sponging miR-206 in ovarian cancer. Cell Physiol Biochem 2018; 49: 1289-1303. https://doi.org//10.1159/000493408

[37] ZHU Y, ZHANG X, QI L, CAI Y, YANG P et al. HULC long noncoding RNA silencing suppresses angiogenesis by regulating ESM-1 via the PI3K/Akt/mTOR signaling pathway in human gliomas. Oncotarget 2016; 7: 14429-14440. https:// doi.org//10.18632/oncotarget.7418

[38] ZHANG X, CHENG SL, BIAN K, WANG L, ZHANG X et al. MicroRNA-26a promotes anoikis in human hepatocellular carcinoma cells by targeting alpha5 integrin. Oncotarget 2015; 6: 2277-2289. https://doi.org//10.18632/oncotarget.2956
[39] LI N, MENG D, GAO L, XU Y, LIU P et al. Overexpression of HOTAIR leads to radioresistance of human cervical cancer via promoting HIF-1a expression. Radiat Oncol 2018; 13: 210. https://doi.org//10.1186/s13014-018-1153-4

[40] ZILLHARDT M, PARK SM, ROMERO IL, SAWADA K, MONTAG A et al. Foretinib (GSK1363089), an orally available multikinase inhibitor of c-Met and VEGFR-2, blocks proliferation, induces anoikis, and impairs ovarian cancer metastasis. Clin Cancer Res 2011; 17: 4042-4051. https://doi. org//10.1158/1078-0432.CCR-10-3387

[41] ZHANG M, JANG H, NUSSINOV R. PI3K inhibitors: review and new strategies. Chem Sci 2020; 11: 5855-5865. https://doi.org//10.1039/d0sc01676d 